\title{
Extraction of Separation Manifolds using Topological Structures in Flow Cross Sections
}

\author{
Alexander Wiebel $^{1}$, Xavier Tricoche ${ }^{2}$, and Gerik Scheuermann ${ }^{1}$ \\ 1 Institut für Informatik, Universität Leipzig \\ \{wiebel|scheuermann\}@informatik. uni-leipzig.de \\ 2 Scientific Computing and Imaging Institute, University of Utah \\ tricoche@sci.utah.edu
}

Summary: The study of flow separation from walls or solid objects is still an active research area in the fluid dynamics and flow visualization communities and many open questions remain. This paper aims at introducing a new method for the extraction of separation manifolds originating from separation lines. We address the problem from the flow visualization side by investigating features in flow cross sections around separation lines. We use the topological signature of the separation in these sections, in particular the presence of saddle points and their separatrices, as a guide to initiate the construction of the separation manifolds. Having this first part we use well known stream surface construction methods to propagate the surface further into the flow. Additionally, we discuss some lessons learned in the course of our experimentation with well known and new ideas for the extraction of separation lines.

\section{Introduction}

In the panel "Even more theory, or more practical applications to particular problems: In which direction will Topology-Based Flow Visualization go?" of the previous workshop (TopoInVis 2005) the (still unsolved) question of benchmark problems arose. These problems should be used to measure the success of the visualization community and more specifically the success of topological methods. The problem posed by one of the participants was the following:

"Given a set of data, say of an ICE train, develop a visualization software which is capable to produce these type of Schlichting ${ }^{1}$ flow visualizations including the stream surfaces separating from the body, including the separation points, vortex core lines and the things which

\footnotetext{
${ }^{1}$ The speaker refers to drawings in a major textbook for fluid dynamics by Schlichting [9]. See Fig. 1 for an example.
} 
you find in the textbook. [...] it would be really interesting if you could do it in an automatic manner.".

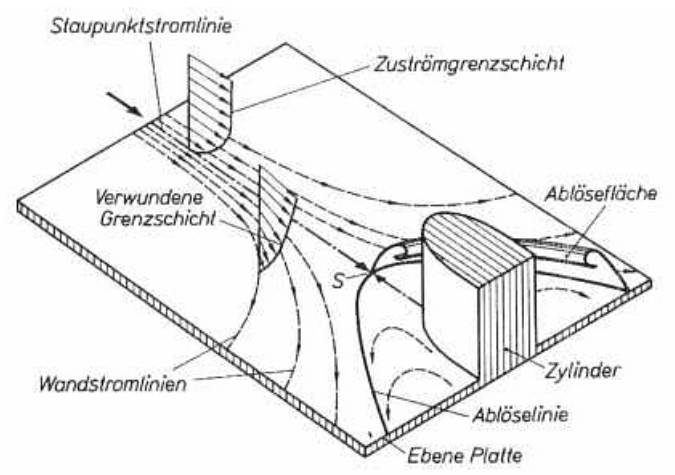

Fig. 1. Drawing of separation line (denoted as Ablöselinie) and separation manifold (denoted as Ablösefläche). Image reprinted from Schlichting [9].

The fact that this problem was posed as a benchmark problem by a fluid dynamics engineer shows that, despite much research and publications on this topic (see Sec. 2), automatic vortex and separation feature visualization are still problems that lack a satisfactory solution. The separation part of this problem provides the motivation for this paper: we aim at automatically extracting separating stream surfaces, one of the building blocks of the type of representations we just mentioned.

The first and simplest idea for the extraction of separation surfaces is to use previously computed separation lines, i.e. connected locations of flow separation, as seeding curves to integrate stream surfaces in the three-dimensional flow. As we will describe later in the paper, this idea and even some more advanced techniques have limitations. This led us to the development of the method presented in this paper. Namely, we compute the flow projection on cross sections along a separation line, construct the topological skeleton of the resulting planar vector fields, and use the saddle point which appears at the separation locus as a guide for the seeding of the separation manifold near the boundary. Having this first part of the surface we employ a standard stream surface integration scheme to compute the remaining part of the surface.

The contents of this paper are organized as follows. We discuss previous work in section 2. The computation of the topological signature of the separated flow is described in section 3 . We then provide results for a variety of realistic CFD problems in section 4. Finally, we conclude our presentation by pointing out topics of future research in section 5 . 


\section{Related Work}

As mentioned in the introduction, flow separation has been a topic of central interest for both the theoretical and experimental sides of fluid dynamics research since the 1950's. A complete overview of the topic is clearly beyond the scope of this paper. Therefore we refer the interested reader to $[2,10]$ and the references therein. Délery [2] described a variety of three-dimensional topological configurations corresponding to separated flows in the smooth setting $^{2}$ and associates them with similar experimental visualizations obtained in a wind tunnel. In a very recent paper Surana et al. [10] proposed a formal theory connecting the Navier-Stokes equations to a topological characterization of separation lines and associated 2-manifolds. The same authors also applied their approach to numerical models computed over simple geometries in [11]. Unfortunately the topological characterization advocated by these authors fails to extract the separation lines and manifolds present in CFD flows defined over more complex polygonal geometries if only the spatial velocity and vorticity data is provided and no access to the simulation is possible (precomputed data). In this case it may be impossible to locate the saddles needed for three of the four separation types mentioned by Surana et al. [10]. This is especially true for the delta wing example they mention. A higher order saddle (or several very close saddles) has to exist at the tip of the wing to serve as origin for separation lines. It strongly depends on the discretization (at the pointed tip) whether such a saddle can be found. Additionally, the skin friction field derived from the precomputed data may be of minor quality prohibiting the use of the formulas reported by Surana et al. [10].

From the visualization viewpoint, the extraction of line-type flow features has motivated a significant body of research in recent years. In addition to the definition and computation of vortex core lines (see [8] for a bibliography), several authors have attempted to detect and display separation and attachment lines on surfaces. The major contribution in this field was made by Kenwright [6] who proposed a method that looks for the presence of separation lines on a cell-wise basis. Upon the assumption of local linearity of the flow a section of separation line is extracted in each triangle as the intersection of specific lines present in the phase portrait of a first-order critical point with the interior of the cell. The discontinuity across cell edges yields disconnected line segments, which was addressed in a subsequent paper [7]. Making the observation that the criterion used in the original method is in fact equivalent to that of zero streamline curvature the authors reformulated their extraction algorithm as the computation of isolines of the flow curvature field. The resulting curves must be filtered in a post-processing step to discard false positives (see [8]). Moreover, the requirement of zero curvature is far too restrictive to account for the general flow geometry associated with flow

\footnotetext{
${ }^{2}$ Here "smooth setting" means the opposite of "discrete setting" like for vector fields from CFD simulations.
} 
separation or attachment. In fact, this criterion was basically tailored to the special case of delta wings, for which separation and attachment lines describe almost straight lines linking the tip of the wing to its back edge. Based on this observation Tricoche et al. [13] recently proposed a method combining a local predictor (the flow divergence) and a global corrector (the one-dimensional convergence behavior of streamlines) to provide a more flexible and robust extraction mechanism. Yet, the corresponding method requires the integration of many streamlines to ensure reliable detection of flow convergence and the filtering of false positives remains a non-trivial and error-prone task. Concerning the use of cross-flow sections or cutting planes Tricoche et al. [12] and Wu et al. [14] proposed methods related to ours. Tricoche uses the cutting planes for general flow visualization problems on special application data sets while $\mathrm{Wu}$ discusses cross sections explicitly in the field of flow separation from the fluid dynamics side.

\section{Extracting Separation Manifolds}

In this section we propose an approach for seeding stream surfaces representing sheets that divide the flow at separation and attachment lines, i.e. separation manifolds. First, we discuss the problems usually arising when trying to compute such surfaces then we give possible solutions to the problems and finally we describe the basic procedures involved in our solution.

\subsection{Seeding Stream Surfaces}

Stream surfaces are well known from the literature [4] and good implementations [3] are readily available for visualization of vector fields. The quality of a stream surface or even the possibility to compute it, however, strongly depends on the availability of a good seed curve for the integration of the dense set of streamlines spanning the surface. The first and obvious idea to get such line strips is to use the extracted feature lines. Unfortunately, this is only a good idea at first sight, because the integration of streamlines in this case starts directly from the surface. This poses problems resulting from the discretization of the surface with polygons and from numerical inaccuracies. The first problem means that if we start streamlines directly from the surface, the integration may yield steps that lead out of the grid as the surface has discontinuities at polygon edges and is not smooth. The second problem is that vertices of feature lines may lie on the wrong side of the surface polygon, i.e. outside of the grid, because of small numerical round-off added while computing the line segments.

Our first attempt to solve these problems consisted in moving the feature lines by a small distance in the direction of the surface normal in order to avoid the issues caused by the irregularities of the polygonal surface while remaining in its immediate vicinity. When we started stream surfaces from the shifted 
lines the integration ran well, but the surfaces, in some cases, did not capture the separation manifold correctly. This is due to the fact that the separation need not happen perpendicular to the surface. Therefore, this method requires knowledge of the angle of the separation in relation to the surface normal. Assuming that this information is available for a point on a feature line we would be able to determine the correct offset vector for this point. We found a formula to do this in the previously mentioned paper of Surana et al. [10]. However, we chose an alternative solution that we found simpler and for our discrete numerical data more appropriate ${ }^{3}$ : we chose to compute the topology of the projected flow in cutting planes along the feature lines and to use the separatrix indicating the separation as guide for the movement of our feature lines or, more precisely, the generation of new starting line strips. Near the surface, the angle between the separation and the surface normal is the same as the angle of this separatrix. The following subsection will describe this idea in more detail.

\subsection{Moving Cutting Planes}

Cutting planes, a very basic and widely used technique, gain more informational value if they are moved along interesting curves (see Fig. 4a). In our case, the type of curves to be used is inherent in the idea of using moving cutting planes. The separation lines provide the natural paths for the moving planes but also leave several degrees of freedom for the orientation of the plane.

\section{Plane Orientation}

The orientation of the plane is a critical parameter of our flow exploration technique because the topological structure in the plane can change dramatically when changing the orientation of the plane. Even when keeping the center position of the plane constant, features can appear or disappear for different orientations of the plane. We would like to use the topology in the plane as a guide for the structure of the flow separation. As the structures which are important for us appear normal to the flow along the separation, we use the surface flow vector at the separation line as first approximation of the normal vector for the cutting plane (see Fig. 4). With this choice, the projected vector field should exhibit a zero vector exactly where the separation line intersects the plane. The zero is a half-saddle with one separatrix leading away from the surface. Near the surface this separatrix indicates the direction of the separation. However, the saddle point can only be found reliably in an analytical setting. As our procedure requires resampling the data and projecting it into the plane, we introduce numerical noise and round-off

\footnotetext{
${ }^{3}$ As mentioned before, we are only working on precomputed data. Thus, not all quantities used in the formula by Surana et al. [10] are available.
} 
error to some extent. This can cause the saddle to move. Lying directly on the boundary of the body, even a small shift can cause the saddle to move out of the plane and thus disappear. Hence, in the case where we do not find a saddle point in the plane we have to adjust its orientation in order to move the desired saddle back onto the plane. Figure 2 shows the basic idea of the adjustment and Figure 3 gives an impression for a real 3D vector field. While we have to tilt the plane against the flow direction for separating flow, we must tilt it with the flow to move the saddle into the plane for attaching flow (see Fig. 2).

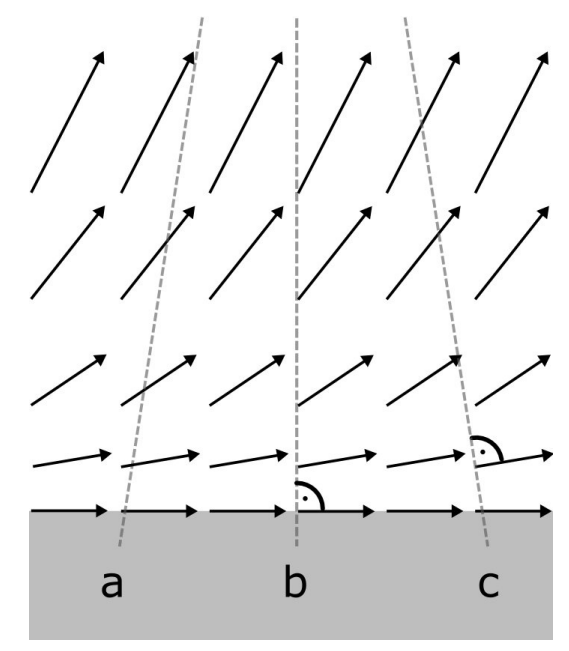

Fig. 2. Saddle moves when tilting plane in separating flow: a) no saddle is present because flow is nowhere perpendicular to plane, b) half-saddle because plane is perpendicular to wall and thus to surface flow, c) saddle moved a small distance away from the wall because plane is perpendicular to separating flow.

We said previously that the orientation of the plane can change the flow dramatically. We have to keep this in mind when tilting the plane, i.e. we have to change the orientation of the plane as little as possible. Changing the inclination of all cutting planes along a line in the same way is the simplest idea but it does not account for the different flow situations at the different base positions along the line. Additionally, such a uniform change would surely not be the smallest change possible for all planes at the same time. Hence, we do not change the inclination uniformly but instead compute the angle for each separate plane through an iterative approach.

As mentioned earlier, we use the velocity as first approximation for the plane normal. To tilt the plane, we turn its normal vector in the plane spanned by the wall normal and the plane normal. This is illustrated in Figure $4 \mathrm{~b}$. 

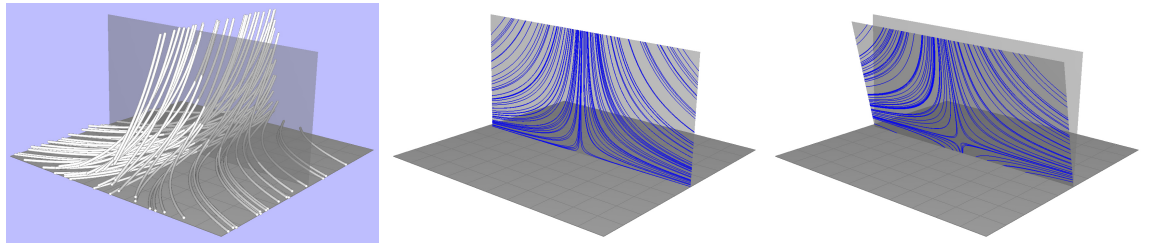

Fig. 3. The left image shows streamlines in a synthetic vector field representing perfect separation and an example for a cutting plane. The image in the middle gives an impression of the projected flow in the plane and the right image shows the flow after tilting the plane. In the middle image only the upper part of the saddle can be seen. The saddle lies exactly on the base plane bounding the flow from below. All sectors of the saddle are observable after it is shifted in the lower image.

The iterative approaching works in two parts. All steps are described in the following:

1. a) Set current angle to a small and constant user prescribed angle.

b) Tilt the original plane by the current angle (against the flow for separation and with the flow in the attachment case).

c) Compute the flow in the tilted plane.

d) Extract the topological structures of the flow.

e) If there is no saddle, decrease the current angle and go back to $1 \mathrm{~b}$.

f) If there is a saddle go to $2 \mathrm{a}$.

2. a) Set $\alpha_{\max }$ to the current angle and $\alpha_{\min }:=0$.

b) Decrease the current angle.

c) Tilt the original plane by the current angle.

d) Compute the flow in the tilted plane.

e) Extract the topological structures of the flow.

f) If there is a saddle set $\alpha_{\max }$ to the current angle.

g) If there is no saddle set $\alpha_{\min }$ to the current angle.

h) Set the current angle value to $0.5\left(\alpha_{\max }+\alpha_{\min }\right)$ and go to $2 \mathrm{c}$.

We iterate for both parts until a maximum number of iterations has been reached. The first part tries to find an initial angle for which a saddle is present on the plane. In the second part of the algorithm, we know that there is a saddle in the plane and try to find the plane with smallest tilting angle and a saddle.

\subsection{Construction of Seed Line for Separation Manifold}

In the previous subsection we described how we compute the topological signature of the separation in the cutting planes. Having this signature, i.e. the separatrices of the saddle point, we can use it to construct the first part of the separation manifold and a start strip for the rest of the surface. At first we have to choose the correct separatrix out of the four separatrices emanating 

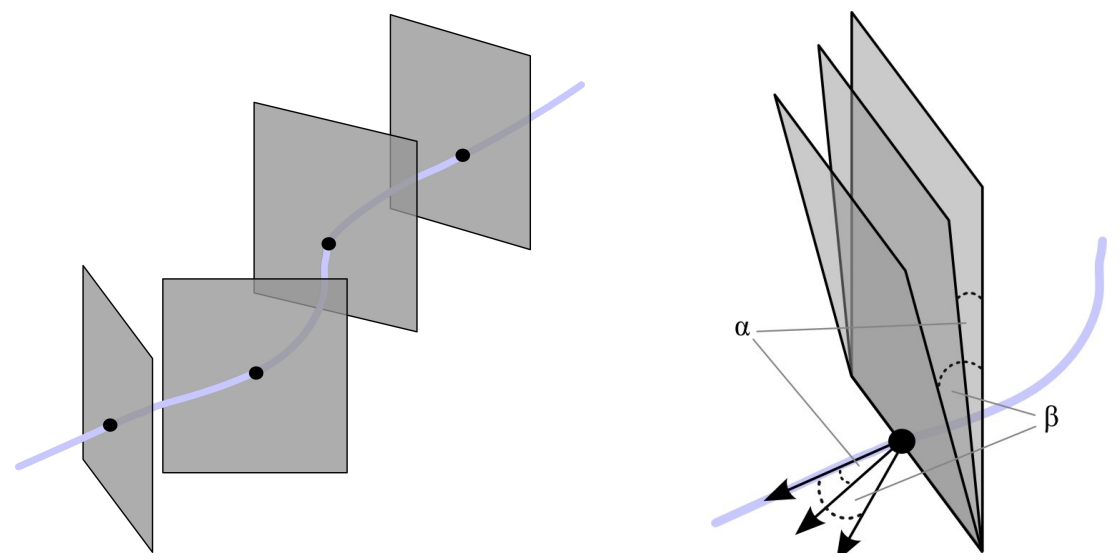

Fig. 4. a) Cutting Planes moving along feature line oriented normal to the flow at their center position. b) Tilting the plane by tilting the normal vector in the plane spanned by the normal vector of the plane and the normal vector of the surface.

from the saddle point. For this task, we take the vector representing the first segment of each of the separatrices and compute its dot product with the body surface normal (all vectors normalized). The segment with the largest value indicates the desired separatrix, as this separatrix is the one which leaves the surface.

To construct the first part of the surface, we take a number of steps along the chosen separatrices in all planes. The step size and the number of steps $m$ can be determined by the user. We then connect the steps of neighboring planes to construct triangles. These triangles build up a surface consisting of $m$ rows of triangles. This surface is the first part of the separation manifold. Splitting the distance for moving along the separatrix into several steps is only important for constructing the triangles. The upper boundary of the last row of triangles, i.e. the connection of the (last) step on all separatrices, constitutes the starting line strip for the rest of the separation manifold.

As mentioned before when discussing the tilting of the plane, it is important to use only structures of the flow in the planes that are very close to the separation line and thus to the body surface. This ensures that the separatrices precisely approximate the structure of the separation manifold.

\section{Results}

In this section we demonstrate our method on two different datasets from CFD simulations. We give a short overview of each dataset, apply our method and discuss the resulting images. 


\subsection{Blunt Fin}

Our first example is a standard reference for flow visualization, the well known blunt fin dataset [5], courtesy of NASA. It represents a steady Mach 2.95 airflow over a flat plate with a blunt fin rising from the plate. The free inflow with a Reynolds number of $2.1 \cdot 10^{6}$ is parallel to the plate and to the flat part of the fin. The dataset represents only one half of the real flow, as it is assumed to be symmetrical about center plane of the fin. In front of the fin two horseshoe vortices coexist with a shock front. The first vortex causes the flow reaching it to separate from the plate. This separation is what we are interested in. The upper image in Figure 5 shows a LIC [1] visualization of the flow over the plate where patterns of converging flow indicate the separation. For the application of our method we extracted a separation line (shown in black) and computed the topological skeleton of 38 section planes moving along the separation line, which took us 2 minutes. We show the section planes to illustrate the procedure. The closeup in the middle left image shows how the separatrices of the saddles in the planes indicate the separation.

The middle right and the lower images show a red surface representing the separation manifold we constructed from the cutting planes. In the right image it is combined with the separatrices of the saddles in the cutting planes. The separatrices are tangential to the surface near the saddle point but intersect it when the distance to the saddle increases. This supports what we mentioned before: the separatrices are good guides for the surface construction but only very close to the saddle. Finally, streamlines in the lower image prove that we computed the correct stream surface, i.e. the one representing the separation manifold.

\begin{tabular}{|l|r|r|r|}
\hline & minimum & maximum & average \\
\hline Angle $\left(^{\circ}\right)$ & 0.006 & 0.27 & 0.026 \\
Distance & 0 & 0.008 & 0.0016 \\
\hline
\end{tabular}

Table 1. Final angle of plane and distance of saddle to surface. Note: size of data set is approx. 10 units.

\subsection{ICE High Speed Train}

The second dataset is a more practically relevant example. It is the high-speed $(I C E)$ train already mentioned in the introduction. This dataset is the result of a simulation of the train traveling at a velocity of about $250 \mathrm{~km} / \mathrm{h}$ with wind blowing from the side at an angle of 30 degrees. The wind causes vortices to form on the lee side of the train, creating a drop in pressure that has adverse effects on the train's track holding. The vortices and the flow passing over the top of the train lead to separating flow at the upper angle on the lee side. 
The separation line we extracted and the flow structure in the corresponding cutting planes is shown in Figure 6. Note how there are no saddles along the blue line where the line does not capture the location of separation correctly. We observed this also in cases where the separation becomes very weak or fuzzy along a line. Streamlines, the separation line, the separation surface we seeded using the cutting planes, and a LIC on the surface of the train give a picture of the complete situation in the lower image. The causal connection between the separation and the vortex formation becomes obvious in this image.

The computations for the 100 cutting planes took 12 minutes.

\begin{tabular}{|l|r|r|r|}
\hline & minimum & maximum & average \\
\hline Angle $\left(^{\circ}\right)$ & $<10^{-8}$ & 3.26 & 0.60 \\
Distance (mm) & 0 & 82.04 & 16.75 \\
\hline
\end{tabular}

Table 2. Final angle of plane and distance of saddle to surface. Note: length of train is approx. $5 \cdot 10^{4} \mathrm{~mm}$

\section{Conclusion}

We have presented a method for automatic computation of separation manifolds from separation lines on bodies immersed in a flow. The method constructs and uses flow cross-sections and the topological signature of the separation therein to construct the surface section in the direct vicinity of the boundary. The construction of the remaining part of the surface relies on standard techniques. Our method proved its usefulness and robustness through application to several different CFD datasets.

For datasets with more intricate flow structures than those presented in this paper, e.g. flows with a very small separation angle, we found problems with the cross-flow in planes very close to the boundary. Thus, our future work will include close collaboration with engineers in order to inspect what is happening near the boundary in these simulations, to try to understand what the problems are and how we can improve our method to handle these problems. As mentioned previously the quality of the surfaces representing the separating flow strongly depends on the accuracy of previously extracted separation lines. Research in the direction of separation line extraction is thus still necessary and will be part of our work until extraction can be performed efficiently and reliably. 


\section{Acknowledgments}

The authors wish to thank Markus Rütten from DLR Göttingen for providing the ICE dataset. Further we are thankful to all members of the FAnToM development team for their programming efforts. This work was partly supported by DFG grant SCHE 663/3-7.

\section{References}

1. Brian Cabral and Leith Casey Leedom. Imaging Vector Fields Using Line Integral Convolution. In SIGGRAPH '93: Proceedings of the 20th Annual Conference on Computer Graphics and Interactive Techniques, pages 263-270, New York, NY, USA, 1993. ACM Press.

2. Jean M. Délery. Robert Legendre and Henri Werlé: Toward the Elucidation of Three-Dimensional Separation. Ann. Rev. Fluid Mechanics, 33:129 - 154, 2001.

3. Christoph Garth, Xavier Tricoche, Tobias Salzbrunn, Tom Bobach, and Gerik Scheuermann. Surface Techniques for Vortex Visualization. In Oliver Deussen, Charles Hansen, Daniel A. Keim, and Dietmar Saupe, editors, Data Visualization 2004 - Eurographics/IEEE TCVG Symposium on Visualization Proceedings, pages 155 - 164, Konstanz, Germany, May 2004. Eurographics Association.

4. Jeffrey P. M. Hultquist. Constructing Stream Surfaces in Steady 3D Vector Fields. In Arie E. Kaufman and Gregory M. Nielson, editors, Proceedings of the 3rd conference on Visualization '92, pages 171 - 178, Boston, MA, 1992.

5. C.M. Hung and P.G. Buning. Simulation of blunt-fin induced shock wave and turbulent boundary layer separation, AIAA Paper 84-0457. In AIAA Aerospace Sciences Conference, Reno, NV, January 1984. AIAA.

6. D. N. Kenwright. Automatic detection of open and closed separation and attachment lines. In IEEE Computer Society Press, editor, IEEE Visualization Proceedings, pages 151-158, Los Alamitos, CA, 1998.

7. D. N. Kenwright, C. Henze, and C. Levit. Features extraction of separation and attachment lines. IEEE Transactions on Visualization and Computer Graphics, 5(2):135-144, 1999.

8. M. Roth. Automatic Extraction of Vortex Core Lines and Other Line-Type Features for Scientific Visualization. PhD thesis, ETH Zrich, 2000.

9. Herrmann Schlichting. Grenzschicht-Theorie. Braun, Karlsruhe, 1992.

10. A. Surana, O. Grunberg, and G. Haller. Exact Theory of Three-dimensional Flow Separation. Part I: Steady Separation. J. Fluid Mech., 564:57 - 103, 2006.

11. A. Surana, G.B. Jacobs, and G. Haller. Extraction of Separation and Reattachment Surfaces from 3D Steady Shear Flows. AIAA Journal, in press, 2006.

12. Xavier Tricoche, Christoph Garth, Gordon Kindlmann, Eduard Deines, Gerik Scheuermann, Markus Ruetten, and Charles Hansen. Visualization of Intricate Flow Structures for Vortex Breakdown Analysis. In Holly Rushmeier, Greg Turk, and Jarke J. van Wijk, editors, Proceedings of the IEEE Visualization 2004 (VIS'04), pages 187 - 194. IEEE Computer Society, October 2004.

13. Xavier Tricoche, Christoph Garth, and Gerik Scheuermann. Fast and robust extraction of separation line features. In Scientific Visualization: The Visual Extraction of Knowledge from Data, pages 245-263. Mathematics + Visualization, Springer, 2005. 
14. J. Z. Wu, R. W. Tramel, F. L. Zhu, and X. Y. Yin. A vorticity dynamics theory of three-dimensional flow separation. Physics of Fluids, 12:1932-1954, August 2000 . 

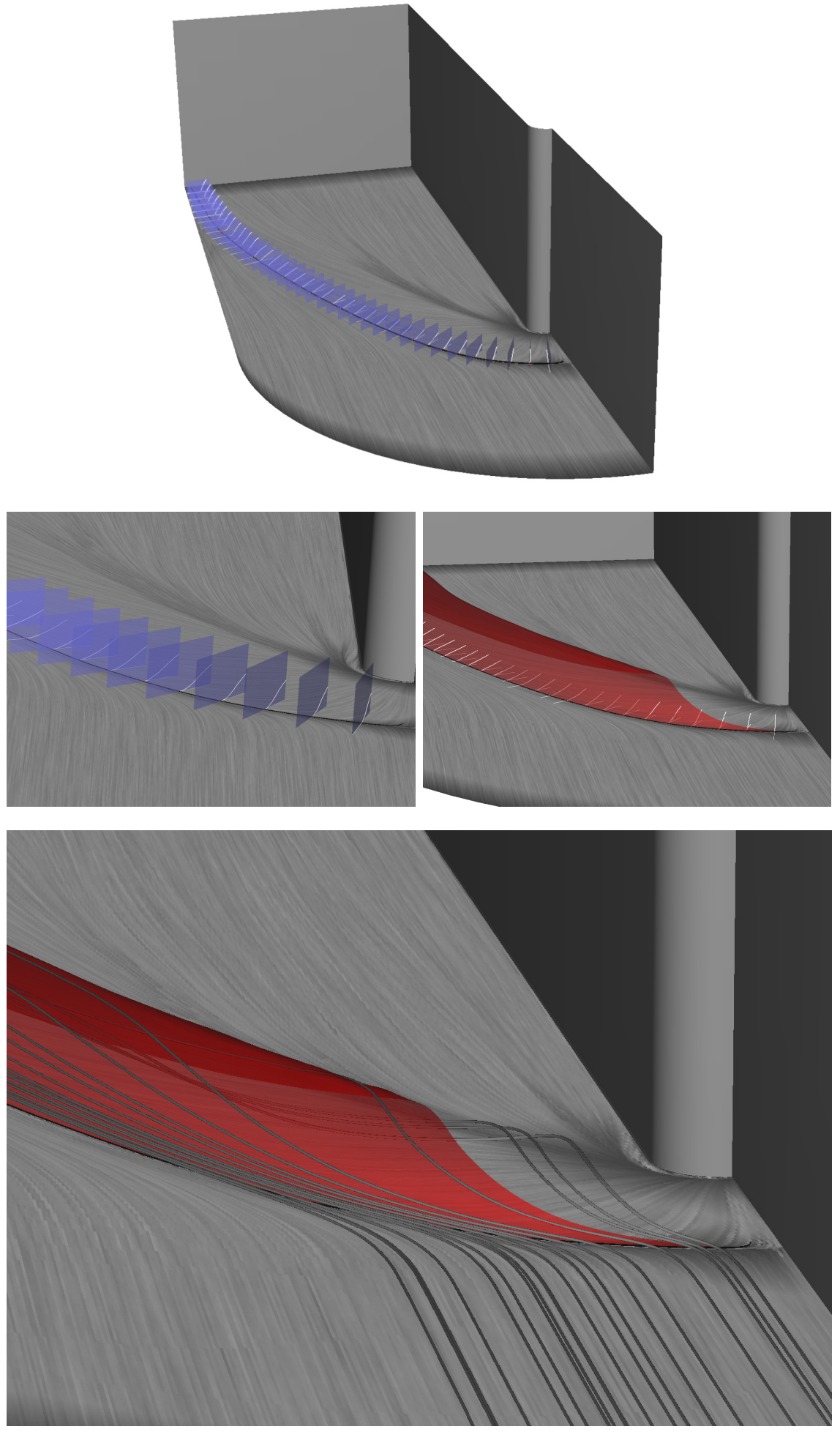

Fig. 5. Blunt fin dataset: The upper image gives an overview of the dataset, the separation line and a number of cutting planes with their topological skeletons. A close-up of the same setting is provided in the middle left image. The middle right and the lower images show the separation manifold constructed from the saddles. In the middle right image the surface is compared with the separatrices in the cutting planes. Streamlines in the lower image prove that the extracted surface is indeed the separation manifold. 

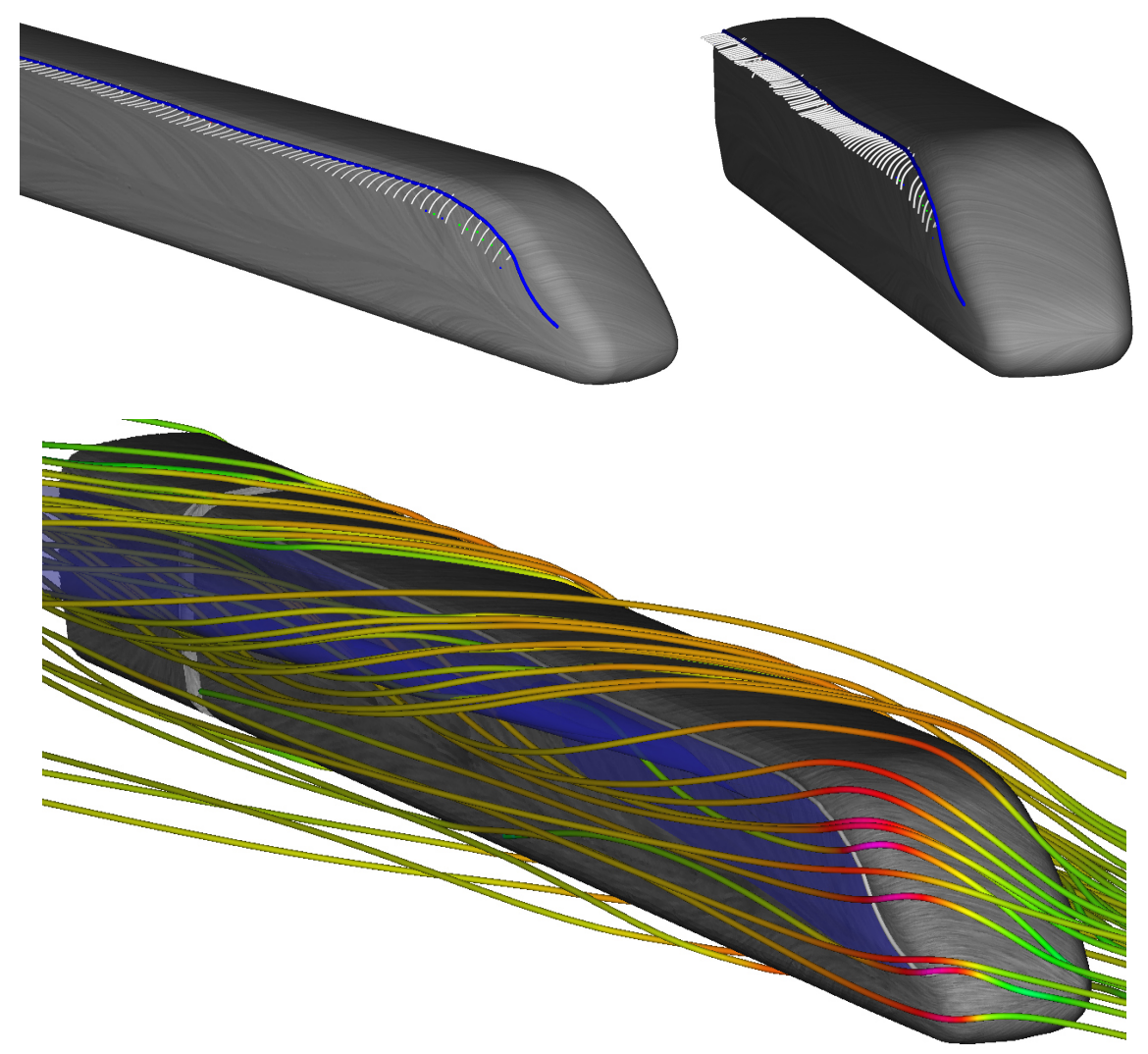

Fig. 6. The upper images show the surface of the ICE train with one separation line and the topological skeletons of about 100 cutting planes along the separation line. Blue and green points mark sinks and source in the cutting planes. The lower image shows a separation manifold generated from the guides in the cutting planes. We additionally provide streamlines to prove that we found the correct separation manifold. 